TITLE:

\title{
Scale effect in the anisotropic deformation change of tracheid cells during water adsorption
}

$\operatorname{AUTHOR}(S)$ :

Taguchi, Ayako; Murata, Koji; Nakamura, Masashi; Nakano, Takato

\section{CITATION:}

Taguchi, Ayako ...[et al]. Scale effect in the anisotropic deformation change of tracheid cells during water adsorption. Holzforschung 2011, 65(2): 253-256

ISSUE DATE:

2011-03

URL:

http://hdl.handle.net/2433/141948

RIGHT:

(c) 2007-2011. Walter de Gruyter GmbH \& Co. KG.; 許諾条件により本文は 2012-04-01に公開 


\section{Scale effect in the anisotropic deformation change of tracheid cells during water adsorption}

\author{
Ayako Taguchi, Koji Murata, Masashi Nakamura \\ and Takato Nakano* \\ Laboratory of Biomaterials Design, Division of Forest \\ and Biomaterials Science, Graduate School of Agriculture, \\ Kyoto University, Kyoto, Japan \\ *Corresponding author. \\ Laboratory of Biomaterials Design, Division of Forest \\ and Biomaterials Science, Graduate School of \\ Agriculture, Kyoto University, Kita-Shirakawa, \\ Kyoto 606-8502, Japan, \\ E-mail: tnakano@kais.kyoto-u.ac.jp
}

\begin{abstract}
The dependence of the deformation change on the location and number of cells during water adsorption was examined by microscopy combined with a "charged-coupled device" camera and image analysis based on the digital image correlation method. The deformation change ratio (DCR) vs. moisture content (MC) in the radial direction approached linearity with $\mathrm{MC}$ with increasing in number of cells between measurement points in earlywood, whereas its relation in latewood was various such as swelling, swelling after shrinking, or remaining constant with MC. The number of cells between observation points clearly affected the DCR vs. MC, i.e., its relationship had the so-called "scale effect"' that a certain physical property appears clearly with increasing scale. The mechanism of the scale effect differed in the radial and tangential directions.
\end{abstract}

Keywords: anisotropy swelling; cell shape; microscopic observation; scale effect; water adsorption.

\section{Introduction}

Wood is a typical anisotropic biomaterial. Various physical properties of wood are affected by its anisotropic characteristics (Norimoto et al. 1978; Ono and Norimoto 1985), which could be influenced by the interaction between earlywood and latewood (Pentoney 1953), restriction by ray tissues (McIntosh 1957), and differences in microfibril angle in the S2 layer (Nakato 1958, Barber and Meylan 1964).

In a previous paper (Taguchi et al. 2010), we observed the deformation behavior of cell cross-sections of wood blocks during water adsorption using confocal laser-scanning microscopy (CLSM) and found that in the tangential (T) direction, cells swelled linearly with increasing moisture content $(\mathrm{MC})$, whereas in the radial $(\mathrm{R})$ direction, cell deformation varied from cell to cell, and some cells even shrank.
For MC in the range of $8-15 \%$, despite differences in shrinkage and swelling, the $\mathrm{R}$ diameter of the tracheids and lumen showed similar tendencies to those reported by Chafe and Ilic (1992) and Sakagami et al. (2007). These results confirm the well-known swelling anisotropy for intact wood blocks in which $\mathrm{T}_{\mathrm{SW}}>\mathrm{R}_{\mathrm{Sw}}$.

Considering both the variation in cell deformation from cell to cell and the uniform deformation of wood blocks with changes in MC, the number of cells is presumed to affect the overall wood deformation. This is because the deformation of whole wood does not necessarily mean all cells swelling with increasing MC. That is, the so-called scale effect might be related to the overall wood deformation. This study examined the dependence of the deformation change during water adsorption on the location and number of cells and the mechanism is discussed.

\section{Materials and methods}

\section{Specimen preparation}

Samples and their preparation are the same as Taguchi et al. (2010). Wood blocks (yezomatsu, Picea jezoensis Carr) measuring 20 $(\mathrm{T}) \times 20(\mathrm{R}) \times 5 \mathrm{~mm}^{3}(\mathrm{~L})$ were prepared. A transverse surface measuring approximately $10(\mathrm{R}) \times 10(\mathrm{~T}) \mathrm{mm}^{2}$ was cut from each block. The transverse surfaces were smoothed with a sliding microtome after softening by soaking in distilled water under vacuum, and then the specimens were dried slowly to avoid collapse at room temperature.

For drying, the specimens were placed sequentially in a desiccator conditioned at $80 \%, 71 \%, 57 \%, 33 \%$, and $11 \%$ relative humidity (RH). They were kept at each condition for at least a week. In the final step, the specimens were conditioned in the desiccator at $11 \% \mathrm{RH}$ for at least 3 weeks, until the $\mathrm{MC}$ achieved equilibrium. The initial MC before the experiments was approximately $4 \%$. No collapse of samples during conditioning was confirmed by microscopic observations after conditioning. A pair of specimens of similar weights was selected from the four specimens obtained from one block. One was used to measure the MC, and the other was used for microscopic observation.

\section{Microscopic observations}

The deformation change of the transverse surface of cells during the water adsorption process was observed by epi-illumination microscopy (EIM) (Nikon Optiphoto, Tokyo, Japan) equipped with a CCD (charge-coupled device) camera. The magnifications of the objective lens were 10 and 20 times. One specimen was weighed and the other observed microscopically in a self-made environmental chamber at a constant temperature of $23^{\circ} \mathrm{C}$ and a given $\mathrm{RH}$ containing an electric balance and a microscope. The chamber made 

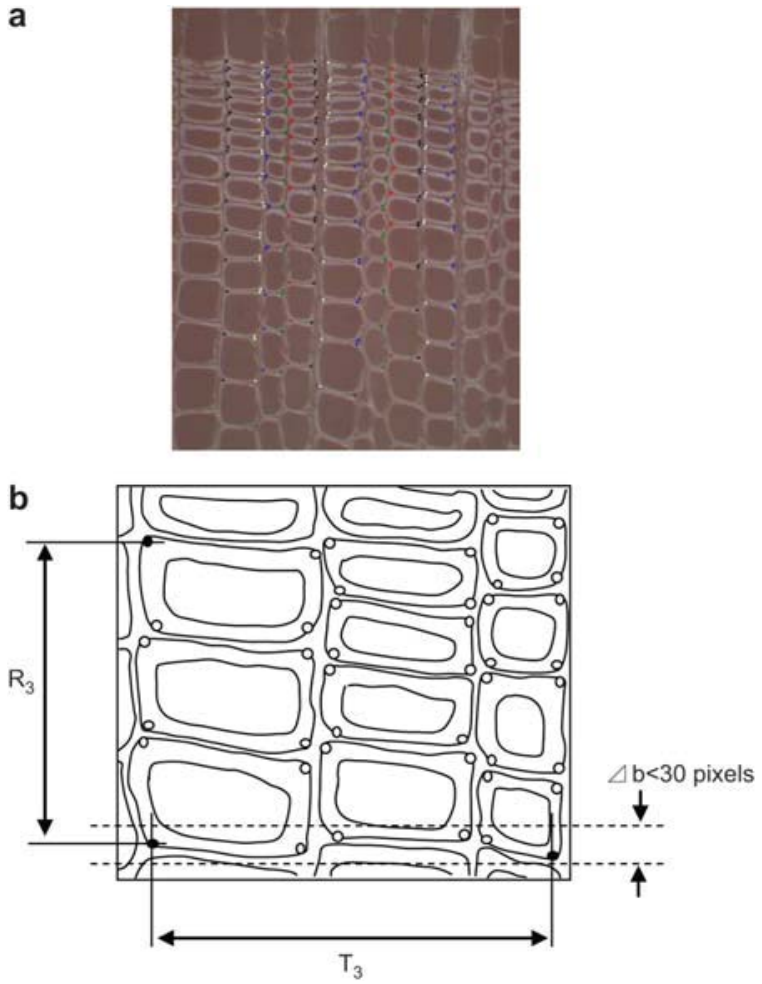

Figure 1 Area observed and the measurement points used in the digital image correlation method. (a) Observation area of a crosssection surface; (b) an example of measurement points being three cells apart.

out of plastic form kept constant temperature and RH during microscopic observation.

First, the specimen was placed in the chamber with silica gel to give a RH of $\sim 30 \%$, and an initial image was obtained. Then, distilled water was placed in the chamber to give a high $\mathrm{RH}$, and images were obtained at regular intervals until the MC approached $10 \%$. Simultaneously, the paired specimen was weighed. During this time, the MC of the specimens increased gradually. Weight measurements were repeated during exposure to moisture. The specimen was oven-dried at $60^{\circ} \mathrm{C}$ under vacuum overnight and weight after observation. $\mathrm{MC}$ was calculated based on the weight differences.

\section{Image analysis}

The digital EIM images measured $1280 \times 1000$ pixels. One pixel corresponded to 0.65 and $0.32 \mu \mathrm{m}^{2}$ for the $10 \times$ and $20 \times$ objection lens, respectively. Twenty-forty images were taken of the observed area on the cross-sectional surface during a water adsorption process. The observed areas were selected in the central area of a digital image without at least 100 pixels from the edge to avoid the effect of aberration (Figure 1a). The observation points were marked at each corner of the cells in a row (Figure 1a,b). Then, the distance between two observation points separated by $1-12$ cells in the $R$ and $\mathrm{T}$ directions was measured during the water adsorption process using the digital image correlation method.

The linearity of the observation points changed minimally in the $\mathrm{R}$ direction during water adsorption because the cells were aligned in a row, whereas this did not necessarily occur in the $\mathrm{T}$ direction. Therefore, the distance between two points within 30 pixels width was measured in the $\mathrm{T}$ direction, as shown in Figure $1 \mathrm{~b}$.

\section{Results and discussion}

The characteristic deformation change of cells in a crosssection of wood blocks was observed during water adsorption by CLSM (Taguchi et al. 2010). In the T direction, the cells swelled linearly with increasing MC, whereas in the R direction, cell deformation varied from cell to cell, and some cells even shrank. Therefore, in the present paper we examined the location dependence of the deformation change in the $\mathrm{R}$ direction.

Figure $2 \mathrm{a}$ shows the typical relationship between $\mathrm{MC}$ and the deformation changes in the $\mathrm{R}$ direction of a cell for four cells; Figure 2b illustrates the observation areas A (LW), B (LW), and $\mathrm{C}(\mathrm{EW})$, where $\mathrm{LW}$ and $\mathrm{EW}$ denote latewood and earlywood, respectively, and $\mathrm{B}(\mathrm{LW})$ is the transition area from LW to EW. The deformation changes are presented in Figure $2 \mathrm{a}$ as a dimensional change rate, calculated as the ratio relative to the initial condition. The changes in region A (LW) involved many deformation modes, from swelling to shrinking. The change appears to approach monotony from LW to EW.

Differences between the linear deformation of the whole wood with MC and the results for each cell presented in Figure $2 \mathrm{a}$ can be interpreted that the deformation depends upon the number of wood cell between measurement points. Therefore, we examined the dependence of the change in deformation on the number of cells in the $\mathrm{R}$ and $\mathrm{T}$ directions.

\section{Radial direction}

Figure $3 \mathrm{a}$ and $\mathrm{b}$ show typical relationships between MC and the deformation change in the $\mathrm{R}$ direction with increasing numbers of cells between the observation points, taken from four type cells. In the LW area, the scatter of the relationship decreased with increasing numbers of cells and disappeared
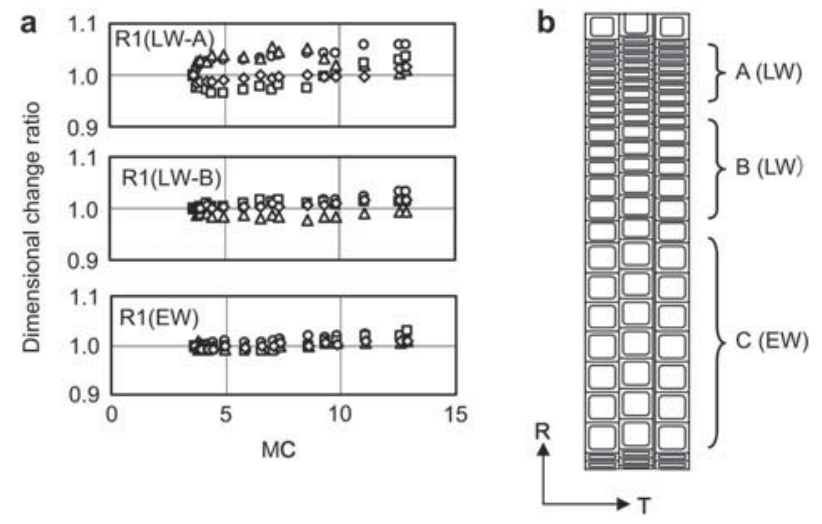

Figure 2 (a) Dimensional change ratios vs. the moisture content (MC) in radial direction (R) in a distance between two observation points of one cell (R1). LW: latewood; EW: earlywood. A, B, and $\mathrm{C}$ refer to the cell regions illustrated on the subfigure (b), i.e., A (LW, latewood), B (LW, transition area in latewood), and $\mathrm{C}$ (EW, earlywood). For example, the symbol R1 (LW-A) indicates the radial change between two observation points one cell apart in latewood area A. The geometrical symbols in the figure are results of four typically different observation areas. 
a
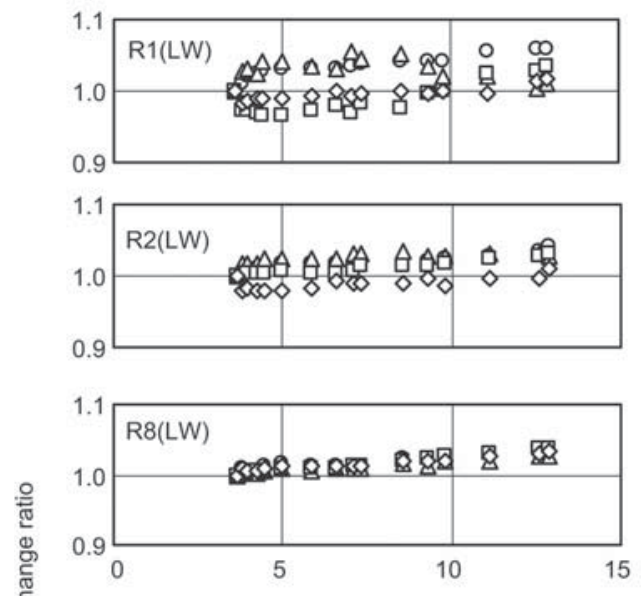

b
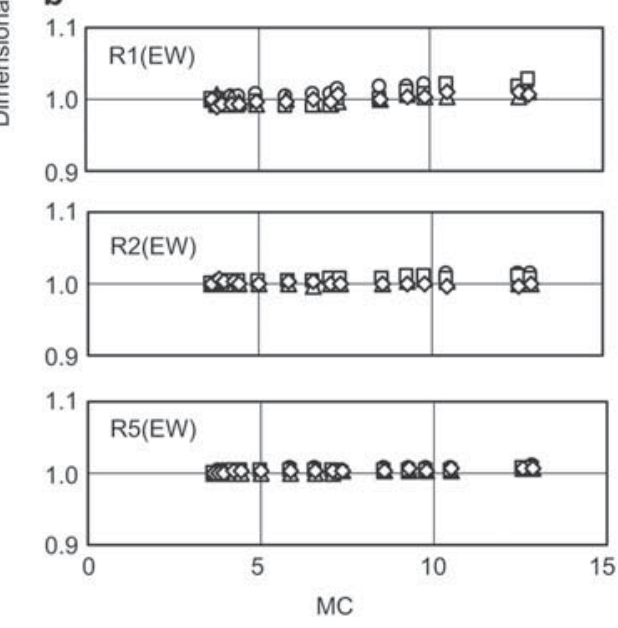

Figure 3 (a) Dimensional change ratios vs. the moisture content (MC) in latewood (LW) in radial direction (R) in a distance between two observation points of one cell (R1), two cells (R2), and eight cells (R8). (b) The same plots for earlywood (EW) in a distance of one cell (R1), two cells (R2), and five cells (R5). The geometrical symbols in the figure are results of four typically different observation areas.

for more than four cells. The dependence on MC also decreased with increasing numbers of cells. By contrast, the effect of the number of cells in the EW area was less than that in the LW. This is because the dependence of the deformation change on the MC in the EW was less than that in the LW, as we demonstrated previously (Taguchi et al. 2010). However, an effect of the number of cells was found even for EW and the slope of the relationship decreased (Figure $3 b)$. The results presented in Figure $3 a$ and b show a systematic dependence in the plots "deformation change ratio vs. MC', which is designated as the so-called scale effect: its effect is that a certain physical property appears clearly with increasing scale.

\section{Tangential direction}

Typical relationships between $\mathrm{MC}$ and the deformation change in the $\mathrm{T}$ direction are shown in Figure $4 \mathrm{a}$ and $\mathrm{b}$ for four cells. The deformation change (as the dimensional change ratio) in the $\mathrm{T}$ direction of a cell changed uniformly with MC (Taguchi et al. 2010). It is visible in Figure 4 that the scatter of the relationship decreased and the slope became even less steep as it was before with increasing numbers of cells in both the LW and EW. That is, the dependence on MC decreased with increasing numbers of cells.

The "scale effect" of the dependence of the deformation change on MC was found in the $\mathrm{T}$ direction, as in the $\mathrm{R}$ direction. However, the mechanism seems to be different in these anatomical directions, as discussed below.

\section{Scale effect on swelling vs. MC}

The scale effect is larger in the $\mathrm{T}$ direction than in the $\mathrm{R}$ direction. Figure 5 could contribute to the discussion of this observation. Here, the slope of the correlation lines (derived from the plots "deformation change vs. MC') is plotted vs.
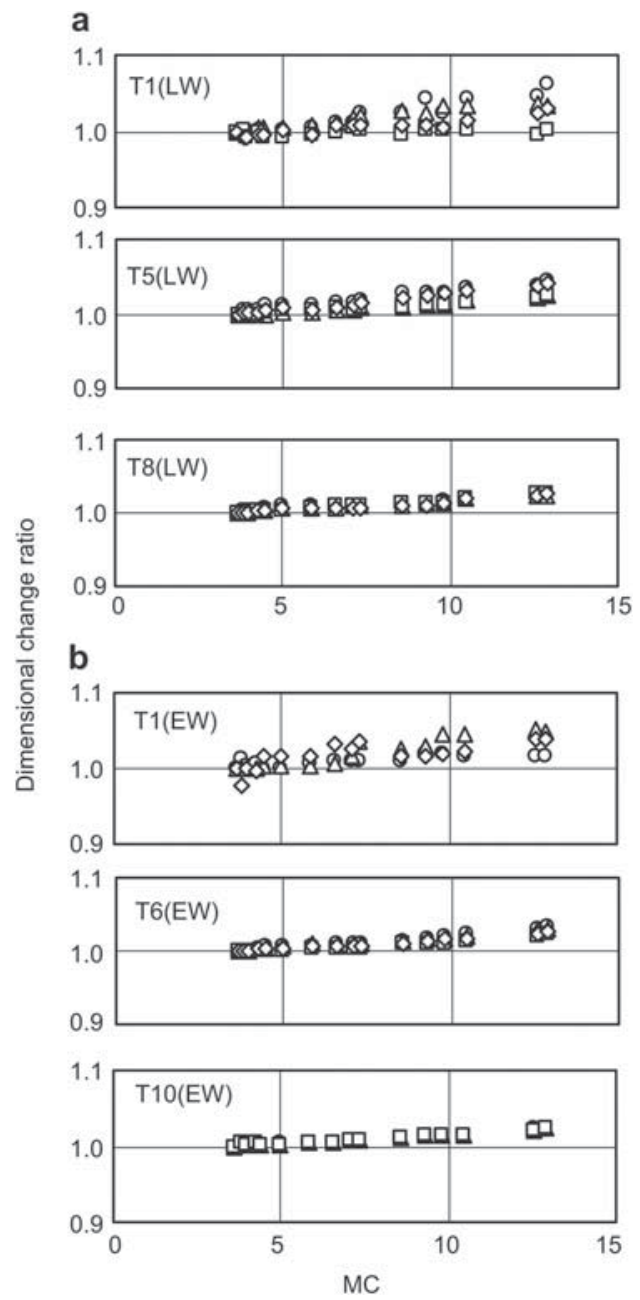

Figure 4 (a) Dimensional change ratios vs. the moisture content (MC) in latewood (LW) in tangential direction (T) in a distance between two observation points of one cell (T1), five cells (T5), and eight cells (T8). (b) The same plots for earlywood (EW) in a distance of one cell (T1), six cells (T6), and ten cells (T10). The geometrical symbols in the figure are results of four typically different observation areas. 


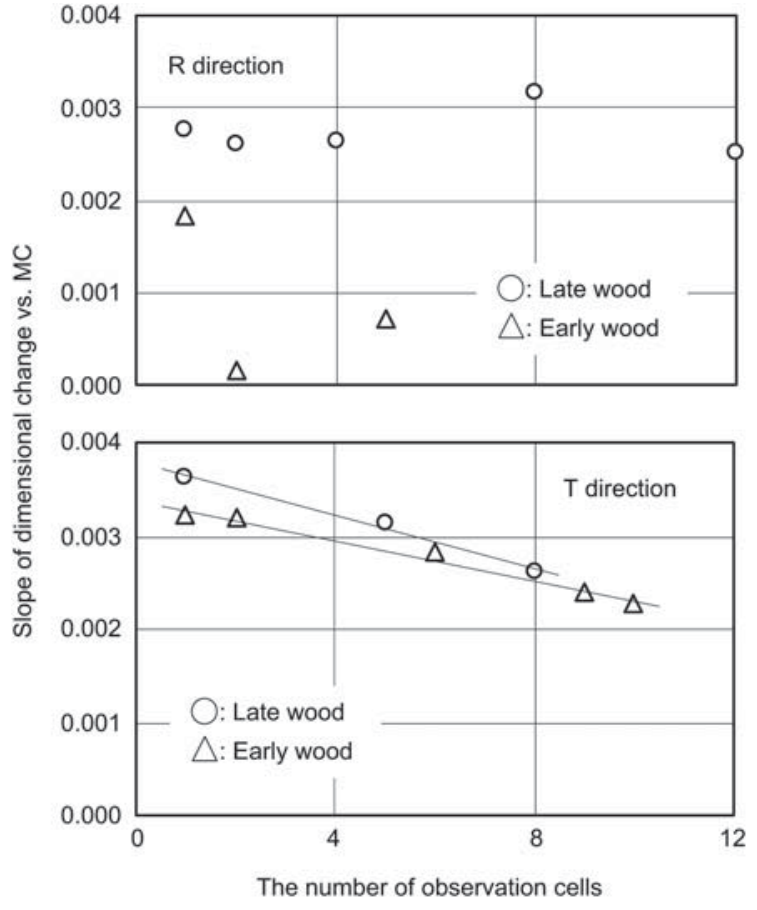

Figure 5 The dependence of the slope of the plots "deformation change ratios vs. MC" on the number of cells between measurement points.

the number of observed cells between the observation points. In the $\mathrm{R}$ direction, the quality of correlation is low. The scattering is due to the different deformation modes - in the range from swelling to shrinking - of the cells under observation. Conversely, the correlation in the $\mathrm{T}$ direction was clear and high. The slope decreased with increasing numbers of cells, i.e., the dependence on MC decreased. Figure 5 could be interpreted only preliminarily, as the data basis for a strict discussion is too little in the present stage of our investigation.

R direction Figure 3 a implies that the dependence of the deformation on the MC was less for an annual ring. This is because the ratio of LW in an annual ring is relatively small in the $\mathrm{R}$ direction. For example, a radial distance of eight cells in area A (LW) was equal to that of five cells in area $\mathrm{B}(\mathrm{LW})$, whereas the radial distance of 12 cells in areas A (LW) and $B(\mathrm{LW})$ was equal to that of five cells in area $C$ (EW), for the observation areas shown in Figure 2b. The deformation change becomes monotonic and the slope decreases as the ratio of $\mathrm{EW}$ increases, because the MC dependence for the EW and the transition area from LW to
EW is monotonic and small. This is the mechanism of the scale effect in the $\mathrm{R}$ direction.

T direction The mechanism in the $\mathrm{T}$ direction differs from that in the $\mathrm{R}$ direction for two reasons. First, a cell row in the $\mathrm{T}$ direction consists of similar cells more uniform than that in the $\mathrm{R}$ direction. As a result, cells in a tangential direction do not undergo various deformation modes, so that the $\mathrm{MC}$ dependent deformation change in the $\mathrm{T}$ direction of a cell is monotonic. Second, the tangential cell row in the $\mathrm{T}$ direction is slightly curved comparing the $\mathrm{R}$ direction cell row. Thus, the distance between two points within 30 pixels width was measured in this study, as illustrated in Figure $1 \mathrm{~b}$. A non-regular row in the $\mathrm{T}$ direction should cause deformation in various directions to some degree. In fact, analysis of the digital images found little cell rotation. This implies that the deformation change of tangential cells contains not only the effects of translation but also of rotation: the effect of cell rotation should be small in the $\mathrm{R}$ direction because of a regular row. However, the effect should be averaged for an increasing number of cells, reducing the scatter.

\section{References}

Barber, N.N., Meylan, B.A. (1964) The anisotropic shrinkage of wood: a theoretical model. Holzforschung 18:146-156.

Chafe, S.C., Ilic, J. (1992) Shrinkage and collapse in thin sections and blocks of Tasmanian mountain ash regrowth. Part 2: The Rratio and changes in cell lumen volume. Wood Sci. Technol. 26:181-187.

McIntosh, D.C. (1957) Transverse shrinkage of red oak and beech. Forest Prod. J. 7:114-120.

Nakato, K. (1958) On the cause of the anisotropic shrinkage and swelling of wood. IX. On the relationship between the microscopic structure and the anisotropic shrinkage in the transverse section (2). Mokuzai Gakkaishi 4:134-141.

Norimoto, M., Hayashi, S., Yamada, T. (1978) Anisotropy of dielectric constant in coniferous wood. Holzforschung 32:167-172.

Ono, T., Norimoto, M. (1985) Anisotropy of dynamic Young's modulus and internal friction in wood. Jpn. J. Appl. Phys. 24: 960-964.

Pentoney, R.E. (1953) Mechanisms affecting tangential vs. radial shrinkage. J. For. Prod. Res. Soc. 3:27-32.

Sakagami, H., Matsumura, J., Oda, K. (2007) Shrinkage of tracheid cells with desorption visualized by confocal laser scanning microscopy. IAWA J. 28:29-37.

Taguchi, A., Murata, K., Nakano, T. (2010) Observation of cell shapes in wood cross-sections during water adsorption by confocal laser-scanning microscope (CLSM). Holzforschung 64: $627-631$.

Received May 27, 2010. Accepted September 20, 2010.

Previously published online November 30, 2010. 